\title{
The Quasar Sdss J153636.22+044127.0: A Double- peaked Emitter in a Candidate Binary Black Hole System
}

\section{Citation}

Tang, Sumin, and Jonathan Grindlay. 2009. "THE QUASAR SDSS J153636.22+044127.0: A DOUBLE-PEAKED EMITTER IN A CANDIDATE BINARY BLACK HOLE SYSTEM." The Astrophysical Journal 704 (2): 1189-94. https://doi.org/10.1088/0004-637x/704/2/1189.

\section{Permanent link}

http://nrs.harvard.edu/urn-3:HUL.InstRepos:41399746

\section{Terms of Use}

This article was downloaded from Harvard University's DASH repository, and is made available under the terms and conditions applicable to Open Access Policy Articles, as set forth at http:// nrs.harvard.edu/urn-3:HUL.InstRepos:dash.current.terms-of-use\#OAP

\section{Share Your Story}

The Harvard community has made this article openly available.

Please share how this access benefits you. Submit a story.

Accessibility 
Draft Version November 8, 2018

Preprint typeset using $\mathrm{LAT}_{\mathrm{E}} \mathrm{X}$ style emulateapj v. 04/20/08

\title{
THE QUASAR SDSS J153636.22+044127.0: A DOUBLE-PEAKED EMITTER IN A CANDIDATE BINARY BLACK-HOLE SYSTEM
}

\author{
Sumin Tang and Jonathan Grindlay \\ Harvard-Smithsonian Center for Astrophysics, 60 Garden St, Cambridge, MA 02138 \\ Draft version November 8, 2018
}

\begin{abstract}
Double-peaked emission lines are believed to be originated from accretion disks around supermassive black holes (SMBHs), and about 3\% of $z<0.33$ AGNs are found to be double-peaked emitters. The quasar SDSS J153636.22+044127.0 has recently been identified with peculiar broad-line emission systems exhibiting multiple redshifts. We decompose the $\mathrm{H} \alpha$ and $\mathrm{H} \beta$ profiles into a circular Keplerian disk line component and other Gaussian components. We propose that the system is both a doublepeaked emitter and a binary SMBH system, where the extra-flux in the blue peaks of the broad lines comes from the region around the secondary black hole. We suggest that such black hole binary systems might also exist in many known double-peaked emitters, where the tidal torques from the secondary black hole clear the outer region of the disk around the primary black hole, similar to the gap in a protostellar disk due to the process of planetary migration, and might also stimulate the formation of a vertical extended source in the inner region around the primary which illuminates the disk. However, most secondary SMBHs in such systems might be too small to maintain a detectable broad line region (BLR), so that the disk line from the primary dominates.
\end{abstract}

Subject headings: quasars: individual (SDSS J153636.22+044127.0) - accretion, accretion disks

\section{INTRODUCTION}

Boroson \& Lauer (2009) recently searched the Sloan Digital Sky Survey (SDSS) archived quasar spectra (Schneider et al. 2007), and identified a quasar, SDSS J153636.22+044127.0 (hereafter SDSS J1536+0441), with two broad-line emission systems separated in velocity by $3500 \mathrm{~km} / \mathrm{s}$. They interpreted it as a sub-parsec supermassive black hole $(\mathrm{SMBH})$ binary, where the two components in the broad emission lines are from the two Broad Line Regions (BLRs) around the pair. The existence of close SMBH binaries are expected as the result of mergers of galaxies, and the sub-parsec scale separation is significant, where the two black holes will eventually coalescence via gravitational radiation (Begelman et al. 1980).

Subsequently, several observations have been carried out to study the system, which also led to other interpretations (Chornock et al. 2009; Wrobel \& Laor 2009; Lauer \& Boroson 2009; Decarli et al. 2009). The most attractive interpretation is as an unusual double-peaked emitter where the multiple components in the broad lines are from the accretion disk around the black hole, which is supported by a third velocity component (a "bump") of the broad emission lines in the red wing (Chornock et al. 2009; Gaskell 2009). Double-peaked emission lines in AGNs have profiles much like the double-peaked emission lines of cataclysmic variables which are due to the disk (Young \& Schneider 1980). However, only about $3 \%$ of $z<0.33$ AGNs are found to be double-peaked emitters in SDSS quasar sample (Strateva et al. 2003), and about 20\% AGNs are found to be double-peaked emitters in a radio-loud AGN survey which includes 106 AGNs (Eracleous \& Halpern 2003). By comparing the spectra taken at different epochs, both Chornock et al. (2009) and Lauer \& Boroson (2009) found no significant velocity evolution of the two main peaks over $\sim 0.7$ years in the rest frame of the object, with a $2-3 \sigma$ upper limit of about $75-80 \mathrm{~km} / \mathrm{s}$. Lauer \& Boroson (2009) showed that the upper limit is still consistent with binary black hole models, and none of the periods longer than $\sim 200$ years has been ruled out.

Both models have their merits, but cannot explain the observed broad line profile well alone. The two shoulders of double-peaked line profiles, which are currently believed to be more likely to come from accretion disks around single black holes (Eracleous et al. 1997), were first suggested to come from two BLRs associated with the black holes of a supermassive binary (Gaskell 1983). However, SDSS J1536+0441 has three velocity components in the $\mathrm{H} \alpha$ and $\mathrm{H} \beta$ profiles, i.e. two sharp peaks and a shoulder-like bump in the red wing, and therefore cannot be explained in the SMBH binary model in Boroson \& Lauer (2009) which only produces two velocity components. On the other hand, the strong sharp blue peaks have no obvious analogue with other doublepeaked emitters and thus require a source other than a normal accretion disk.

However, the two explanations may fit together well. Accretion onto AGN will usually be through an accretion disk, while SMBH binaries are expected as normal consequences of galaxy mergers, therefore combining the two together is reasonable. Here we propose an alternative hypothesis that the system is both a double-peaked emitter and a binary black hole system, where the extra-flux in the blue peaks of the broad lines comes from the region around the secondary black hole, and the disk lines are from the accretion disk around the primary black hole. In this paper, we refer to the Broad Line Region, or BLR, as any region producing the broad lines, which may then include both the disk and discrete clouds. In section 2, we fit the $\mathrm{H} \alpha$ and $\mathrm{H} \beta$ line profiles using a circular Keplerian disk model. In section 3, we describe our model to explain the system. We then discuss in section 
4 that SMBH binary systems might also exist in many other double-peaked emitters.

\section{DISK MODEL FIT}

In about half of the known double-peaked emitters, the broad double-peaked lines could be fit well by a circular, symmetric, relativistic, Keplerian disk (60\% in Eracleous \& Halpern 2003 and $40 \%$ in Strateva et al. 2003), while others require some form of asymmetry (hot spots, elliptical disks, warps, spiral shocks, etc.). Since we do not have any prior knowledge that the disk is asymmetric and if so how it would be, we chose the axisymmetric Keplerian disk model from Chen \& Halpern (1989), which has five parameters: the inclination angle $i$, inner and outer radii $r_{1}$ and $r_{2}$ in units of the gravitational radius $r_{G}$, local broadening of the line which is represented by a Gaussian rest-frame profile of velocity dispersion $\sigma$, and the index of the surface emissivity power law $q$. We fixed $q=-3$, as predicted in photonionization calculations (Collin-Souffrin \& Dumont 1989; Dumont \& CollinSouffrin 1990). For simplicity, we further fixed $\sigma=1200$ $\mathrm{km} / \mathrm{s}$ which is a typical value for double-peaked emitters (see e.g. Strateva et al. 2003).

To physically constrain the fits, we assume that the Narrow Line Region (NLR) is associated with the central component (r-system in Boroson \& Lauer 2009), and all narrow lines are Gaussian with the same Full Width at Half Maximum (FWHM). We also assume that both $\mathrm{H} \alpha$ and $\mathrm{H} \beta$ disk lines are from the same disk, so they must have the same inclination angle. We fitted the $\mathrm{H} \alpha$ and $\mathrm{H} \beta$ lines from Chornock et al. (2009) in the following steps:

1. We first fit a disk line to $\mathrm{H} \alpha$, since it is less blended than $\mathrm{H} \beta$. We subtract the disk line from the spectrum, and fit the residuals with 5 Gaussian profiles: one for the blue peak, two for the [N II] $\lambda 6548$ and 6583 (constrained to 1:3 height ratio), and two (NLR, BLR) for the central peak. We then subtract the 5 Gaussian profiles, and the residuals are considered to be the disk line component, and we re-do the disk line fit to derive the inclination for the disk component.

2. We use the inclination angle derived in step 1 , and fit a disk line to the $\mathrm{H} \beta$ profile. We subtract the disk line from the spectrum, and fit the residual with 5 Gaussian profiles: one for the blue peak, two for [O III] $\lambda 4959$ and 5007 lines respectively, and two (NLR, BLR) for the central peak. The central narrow $\mathrm{H} \beta$ component and the two [O III $] \lambda$ 4959 and 5007 lines are required to have the same FWHM.

3. We then use the FWHM derived in Step 2 to re-fit the $\mathrm{H} \alpha$ profile and require the central narrow $\mathrm{H} \alpha$ component, [N II] $\lambda 6548$ and 6583 (constrained to $1: 3$ height ratio) to have the same FWHM as the [O III] $] 4959$ and 5007 lines (Figure 1).

4. Again, we used the inclination angle derived in step 3 , and re-fit the $\mathrm{H} \beta$ profile (Figure 2).

The fitting results are shown in Figure 1 for $\mathrm{H} \alpha$, Figure 2 for $\mathrm{H} \beta$, and Table 1 for the best-fit parameters

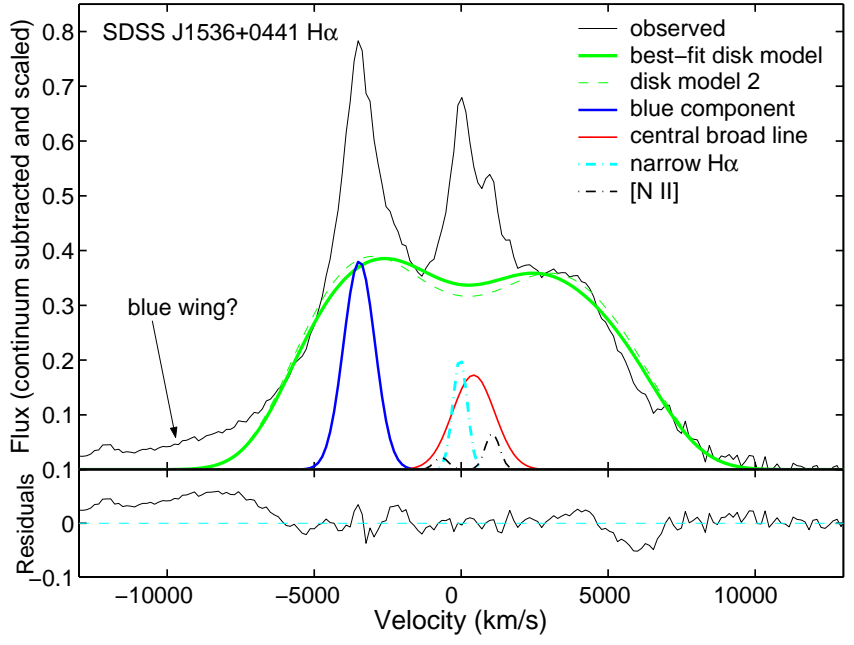

FIG. 1. - H $\alpha$ emission line profile of SDSS J1536+0441. In the upper main panel, the black line is the continuum-removed spectrum from Chornock et al. 2009, the solid green line is the best-fit disk model line, the left blue line is the Gaussian fit to the blue peak, the central red line is the Gaussian fit to the central peak (r-system) with the narrow line component removed, and the cyan dash-dotted line is Gaussian fit to the narrow $H \alpha$, and black dashdotted lines are Gaussian fits to [N II] $\lambda 6548,6583$ lines, with fixed FWHM given by fitting [O III] $\lambda 4959,5007$. The dashed green line shows the disk model line with outer radius $8000 r_{G}$, and the same inner radius and inclination as the best-fit solid green line. The black solid line in the lower panel shows the residuals.

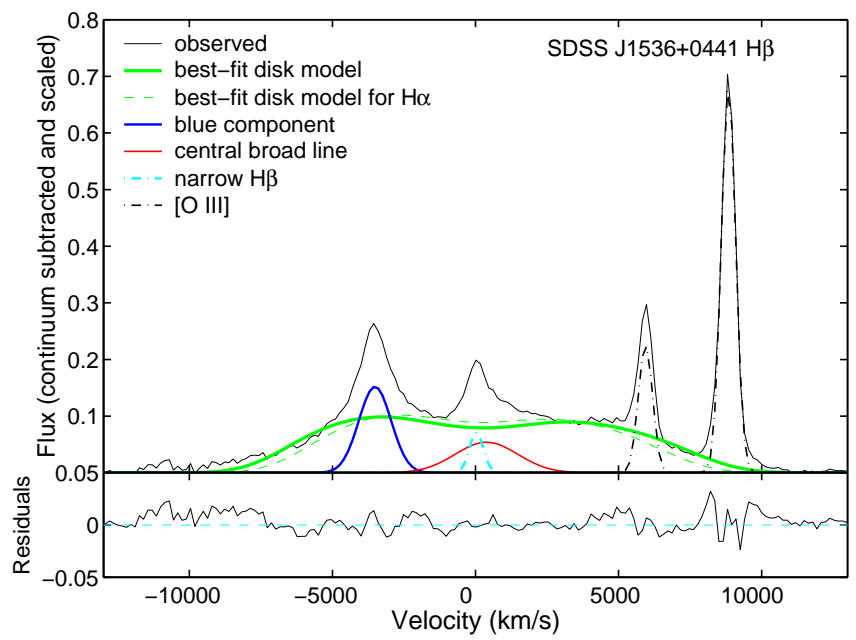

FIG. 2.- H $\beta$ emission line profile of SDSS J1536+0441. In the upper main panel, the black line is the continuum removed spectrum from Chornock et al. 2009, the solid green line shows the best-fit disk model line with fixed inclination given in the fitting of $H \alpha$ profile, the dashed green line shows the best-fit disk model line for $H \alpha$ in Figure 1, the left blue line is the Gaussian fit to the blue peak, the central red line is the Gaussian fit to the central peak with the narrow line component removed, the dash-dotted cyan lines are Gaussian fits to the narrow $H \beta$, and the black dashdotted lines are Gaussian fits to [O III] $\lambda 4959,5007$ lines. The black solid line in the lower panel shows the residuals.

(with least $\chi^{2}$ ). From the best-fit model, the disk inclination is $i \sim 47^{\circ}$. For the $\mathrm{H} \alpha$ profile, the inner radius $r_{1} \sim 1000 r_{G}$, and the outer radius $r_{2} \sim 13000 r_{G}$. For the $\mathrm{H} \beta$ profile, $r_{1} \sim 800 r_{G}$, and $r_{2} \sim 7000 r_{G}$. In principle, the inner disk radius and the inclination determine the width of the line, the inclination could be decoupled from the inner radius from the net Gravitational redshift 
TABLE 1

EMISSION-LINE FIT PROPERTIES OF SDSS J1536+0441.

\begin{tabular}{lllll}
\hline Components & Line $(\AA)$ & Vel. $^{\mathrm{a}}$ & $\mathrm{FWHM}^{\mathrm{b}}$ & Line Ratio $^{\mathrm{c}}$ \\
\hline Double-peaked & $\mathrm{H} \alpha$ & $\mathrm{N} / \mathrm{A}^{\mathrm{d}}$ & 13580 & 8.5 \\
Double-peaked & $\mathrm{H} \beta$ & $\mathrm{N} / \mathrm{A}^{\mathrm{d}}$ & 14890 & 5.7 \\
\hline Blue-system & $\mathrm{H} \alpha$ & -3470 & 1340 & 1.0 \\
Blue-system & $\mathrm{H} \beta$ & -3510 & 1330 & 1.0 \\
\hline Central broad line & $\mathrm{H} \alpha$ & 420 & 1690 & 0.61 \\
Central broad line & $\mathrm{H} \beta$ & 380 & 2570 & 0.69 \\
\hline Narrow lines & $\mathrm{H} \alpha$ & $-\mathrm{e}$ & $573^{\mathrm{f}}$ & 0.24 \\
Narrow lines & {$[\mathrm{N} \mathrm{II}] 6548$} & $-\mathrm{e}$ & $573^{\mathrm{f}}$ & $0.027^{\mathrm{g}}$ \\
Narrow lines & {$[\mathrm{N} \mathrm{II}] 6583$} & $-\mathrm{e}$ & $573^{\mathrm{f}}$ & $0.08^{\mathrm{g}}$ \\
Narrow lines & $\mathrm{H} \beta$ & $-\mathrm{e}$ & $573^{\mathrm{f}}$ & 0.20 \\
Narrow lines & {$[\mathrm{O}$ III] 4959} & $-\mathrm{e}$ & $573^{\mathrm{f}}$ & 0.66 \\
Narrow lines & {$[\mathrm{O}$ III $] 5007$} & $-\mathrm{e}$ & $573^{\mathrm{f}}$ & 2.0 \\
\hline
\end{tabular}

${ }^{a}$ Velocities in $\mathrm{km} / \mathrm{s}$, negative value means blueshifted and positive value means redshifted.

${ }^{\mathrm{b}} \mathrm{FWHM}$ of the lines in $\mathrm{km} / \mathrm{s}$ using the reduced rest-frame spectra in Chornock et al. 2009.

c Line ratios are calculated compared with the blue component, i.e. all the lines in Figure $1(\mathrm{H} \alpha)$ are compared with the line flux of the blue $\mathrm{H} \alpha$ component, and all the lines in Figure $2(\mathrm{H} \beta)$ are compared with the line flux of the blue $\mathrm{H} \beta$ component.

${ }^{d}$ Not available since the centers of the disk model lines are fixed in the fitting.

eThe narrow lines are constrained within $5 \AA$ of their air wavelengths in the fitting, and it turned out all of them are consistent with zero velocity within $95 \%$ confidence level.

${ }^{\mathrm{f}} \mathrm{FWHM}$ of all the narrow lines are constrained to be the same value in the fitting.

${ }^{g}$ [N II] 6548 and 6583 are constrained to 1:3 height ratio.

of the line, and the outer radius determines the separation of the two shoulder-like peaks (Chen \& Halpern 1989; Strateva et al. 2003). However, in the case of SDSS J1536+0441, the shoulder-like peaks are obscured by the blue peaks in both $\mathrm{H} \alpha$ and $\mathrm{H} \beta$, and blended by [O III] $\lambda 4959$ in the $\mathrm{H} \beta$ profile, plus, there is a blue wing component which cannot be fit well by an axisymmetric Keplerian disk model, as shown obviously in the $\mathrm{H} \alpha$ profile and also present in $\mathrm{H} \beta$ but weaker. As a result, the three remaining parameters in the disk model, i.e. the inclination angle and the inner and outer radii, are still degenerate to some degree, as shown in Figure 3, and the errors are hard to estimate. Therefore, we did not attempt to derive the errors in a statistical way, or try to get the absolute value of the $\chi^{2}$ values of the fits. From the $\mathrm{H} \alpha$ profile fits, we cannot really distinguish model lines with inner radius range from $800 r_{G}$ with smaller inclination angle $\left(i \sim 40^{\circ}\right)$ vs $1400 r_{G}$ with larger inclination angle $\left(i \sim 60^{\circ}\right)$, as shown in Figure 3. We also cannot really distinguish model lines with smaller outer radius like $8000 r_{G}$ from model lines with larger outer radius like $13000 r_{G}$, as shown in the dashed line (disk model 2) and solid green line (best-fit model line) in Figure 1 . Therefore, the best-fit parameters are only good as a rough initial estimate.

Nevertheless, there are several very interesting features evident from the circular disk model fitting:

1. As shown in Figures 1-2, both the $\mathrm{H} \alpha$ and $\mathrm{H} \beta$ profiles can be fit reasonably well by a model with 4 components: a simple circular disk line from the accretion disk around a central object (r-system), narrow lines from a NLR associated with the central object (r-system), a central broad line from the

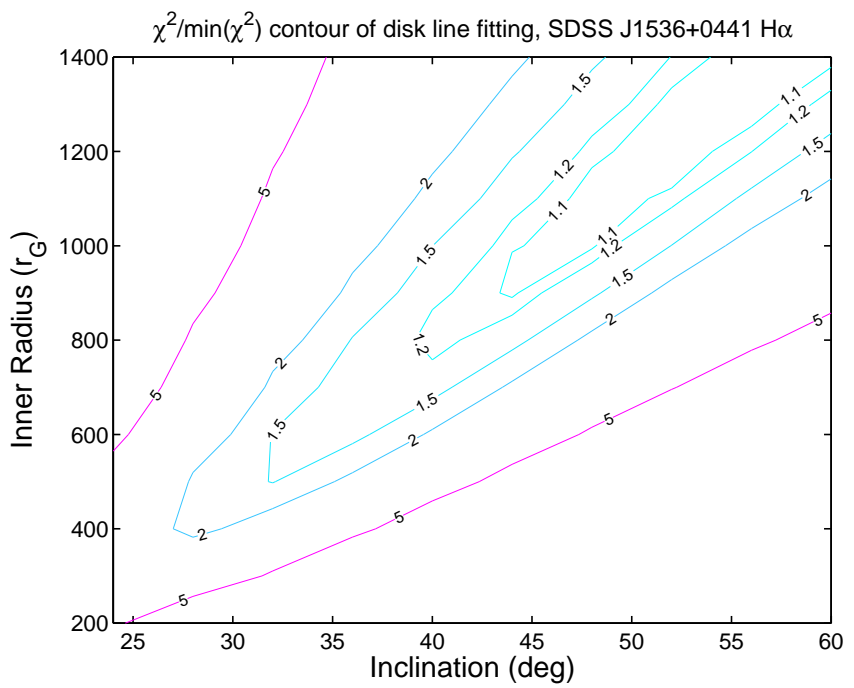

FIG. 3. - Re-scaled- $\chi^{2}$ contour of fittng $\mathrm{H} \alpha$ emission line profile of SDSS J1536+0441 in an axisymmetric Keplerian disk model from Chen \& Halpern (1989). We only computed models with inclination smaller than 60 degree, as is usually the case for type I AGN.

BLR associated with the central object (r-system), and a broad blue line in another velocity field (bsystem). Only the first three components in the r-system are normal in double-peaked emitters.

2. The $\mathrm{H} \beta$ disk line is obviously wider than $\mathrm{H} \alpha$, which means $\mathrm{H} \beta$ is emitted primarily from smaller radii in the disk. As shown in Figure 2, the best-fit disk line for the $\mathrm{H} \beta$ is shown in solid green, while the best-fit disk line for $\mathrm{H} \alpha$ is shown in dashed green. Here $\operatorname{FWHM}(\mathrm{H} \alpha)=0.91 \mathrm{FWHM}(\mathrm{H} \beta)$, which is consistent with the higher excitation temperature for $\mathrm{H} \beta$, and is consistent with the value found in normal (non-double-peaked emitter) AGNs (Stirpe 1990; Kaspi et al. 2000).

3 . The central broad component is redshifted compared with the narrow lines by $\sim 400 \mathrm{~km} / \mathrm{s}$, indicating there might be some form of inflow. Fe II emission and intermediate-width $\mathrm{H} \beta$ components are found in SDSS AGNs to be systematically redshifted by about $400 \mathrm{~km} / \mathrm{s}$ with respect to the narrow lines, which may come from an intermediateline region located at the outer portion of the BLR which is dominated by inflow (Hu et al. 2008a, $2008 \mathrm{~b})$. The existence of such an intermediatewidth broad line region might be common in AGNs (see e.g. Brotherton et al. 1994; Zhu et al. 2009). Another alternative explanation is gravitational redshift. Eracleous \& Halpern (1994) found in a radio-loud AGN sample that the broad $\mathrm{H} \alpha$ lines at half-maximum are preferentially redshifted by $\langle\Delta \lambda / \lambda\rangle=(6 \pm 2) \times 10^{-4}$ on average, i.e. $\sim 180 \mathrm{~km} / \mathrm{s}$. If the $400 \mathrm{~km} / \mathrm{s}$ redshift in the central broad component is caused only by gravitational plus transverse redshift, then it corresponds to Keplerian motion at a radius of $\sim 10^{3} r_{G}$ and a Keplerian velocity of $\sim 10^{4} \mathrm{~km} / \mathrm{s}$, which is much larger than the FWHM of the central broad lines. Therefore, it is more likely come from some form of inflow. 


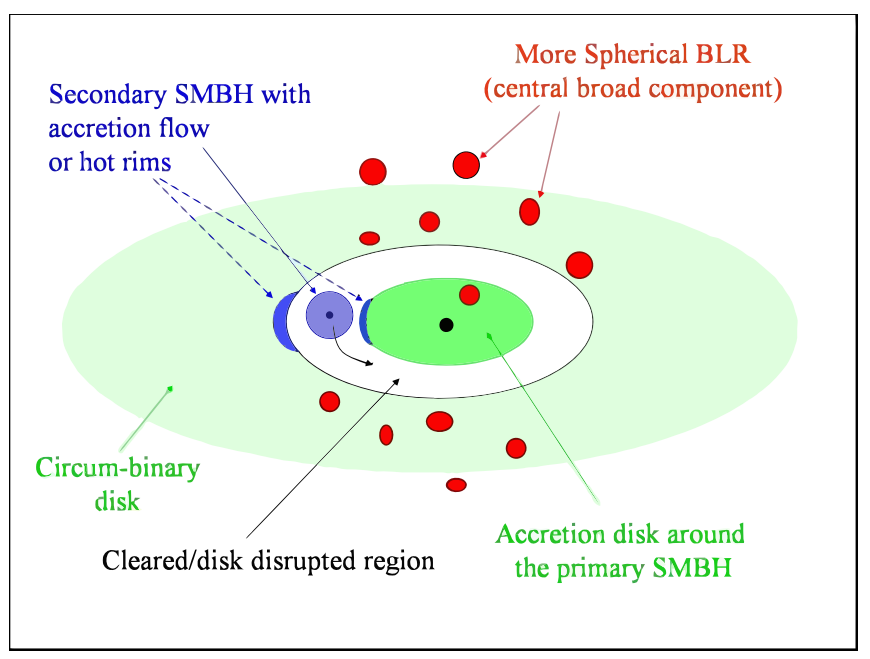

FIG. 4.- Illustration of our model for SDSS J1536+0441, where the secondary SMBH spirals in and clears a gap in the gas disk around the primary SMBH (not drawn to scale). The secondary is currently moving towards us. In the context of the model proposed here, the disk-line component comes from the accretion disk around the primary black hole, the central component comes from the more spherical BLR around the primary black hole, and the blue component comes from either the accretion flow around the secondary SMBH, or the rims of the circum-primary and the circum-binary disks which are close to and heated by the tidal perturbations of the secondary.

4. There is a blue wing component, strong in the $\mathrm{H} \alpha$ profile and weaker in the $\mathrm{H} \beta$ profile, with velocity as high as $\sim 10,000 \mathrm{~km} / \mathrm{s}$ and indicating some form of outflow. Such a component has been seen in many double-peaked emitters (e.g. 3 doublepeaked AGNs out of a sample of 8 in Eracleous \& Halpern 2003, showed a blue wing excess), and could be the wind from the accretion disk (Elvis 2000).

\section{BINARY BLACK HOLE AS A MODEL TO EXPLAIN SDSS $\mathrm{J} 1536+0441$}

As discussed in Lauer \& Boroson (2009), the sharppeaked blue component in SDSS J1536+0441 is different from what is seen in known double-peaked emitters, which are usually more flattened and shoulder-like. This blue component, which was the key characteristic stimulating the study of the system and was discussed extensively in previous papers (Boroson \& Lauer 2009 and references therein), could possibly be fit by an extremely asymmetric disk model, where the blue peak comes from a high emissivity region (such as a hot spot) moving towards us. However, how to produce and maintain such a huge hot spot is not clear.

Instead of invoking a highly-asymmetric disk model, alternatively, we propose that SDSS J1536+0441 is a binary black hole system, where the disk-line component comes from the accretion disk around the primary black hole, the central component comes from the more spherical BLR around the primary SMBH, and the blue component comes from the region around the secondary SMBH, which is moving towards us right now. Our model is illustrated in Figure 4.

The blue peaks could come from the accretion flow onto the secondary SMBH. The possible existence of two broad line systems as the consequence of two merging black holes has been predicted by Gould \& Rix (2000), one from the primary and one from the accretion disk around the secondary. Fe II, which is believed to be photoionized and probably from winds flowing off the surfaces of accretion disks of AGNs (Baldwin et al. 2004; Vestergaard \& Peterson 2005), has been found primarily associated with the blue-system velocity (Boroson \& Lauer 2009; Lauer \& Boroson 2009), and supports the existence of an accretion disk around the secondary.

The blue peaks could also come from the rims of the circum-primary and the circum-binary disks, which are close to and heated by the tidal perturbations of the secondary SMBH. Lodato et al. (2009) showed in their simulations that the tidal torques from the secondary SMBH heat the edges of the gap, creating bright rims around the secondary, which could account for as much as $50 \%$ of the integrated light from the disk. SDSS J1536+0441 would be a perfect example if this is the case. Note the velocity separation between the blue peak and the central peak $(v \sim 3500 \mathrm{~km} / \mathrm{s})$ is similar to the Keplerian velocity of the disk outer radii. If we assume the inclination angle as $i=47^{\circ}$, the separation velocity is the projected orbital velocity of the secondary, and the secondary is just moving towards us, then its orbital radius is $2(c \sin i / v)^{2} \sim 8000 r_{G}$, which is consistent with the outer radius in our $\mathrm{H} \alpha$ (see the disk model 2 (dashed green line) in Figure 1 ) and $\mathrm{H} \beta$ profile fits.

Though there is one double-peaked emitter, 3C 390.3, in the reverberation mapping AGN sample, which follows the Luminosity-BLR size relation (hereafter L-R relation) well (Peterson et al. 2004; Kaspi et al. 2005), it is under debate whether the empirical L-R relation derived from reverberation mapping results could be applied to double-peaked AGNs (Wu \& Liu 2004; Zhang et al. 2007). Zhang et al. (2007) found that the black hole masses of double-peaked emitters estimated from pure stellar velocity dispersion (Lewis \& Eracleous 2006) are about six times smaller than the virial black hole masses using the reverberation mapping L-R relation and FWHMs of $\mathrm{H} \beta$ lines, though in some AGNs the two masses are consistent while in some others the virial mass could be 10-20 times larger. If we assume a typical starlight fraction value of 0.33 for double-peaked emitters (Eracleous \& Halpern 2003), and all the other continuum is from the primary SMBH, then use the FWHM of the best fit double-peaked $\mathrm{H} \beta$ line, we could derive a virial black hole mass following the reverberation mapping L$\mathrm{R}$ relation (Kaspi et al. 2005). We adopt a flat cosmology with $H_{0}=71 \mathrm{~km} / \mathrm{s} / \mathrm{Mpc}$ and $\Omega_{m}=0.27$ ), and find the size of the primary BLR (which is the accretion disk here) $R=141$ lt-days, $M=4.6 \times 10^{9} M_{\odot}$. If we adopt the value of six as the factor of the mass increase by the virial calculation, then the mass of the primary is about $M_{1}=10^{9} M_{\odot}$. It is difficult to estimate the mass of the secondary. As discussed in section 2, the central broad component is redshifted by $\sim 400 \mathrm{~km} / \mathrm{s}$. If it is due to the orbital motion of the primary SMBH rather than inflow, while the blue broad component is due to the orbital motion of the secondary, then the mass ratio will be $\sim 0.1$ and the secondary SMBH mass will be $M_{2}=10^{8} M_{\odot}$. However, this is unlikely to be the case, since the disk line component agrees well with the redshifts of the narrow lines, and therefore the secondary to primary mass ratio must be smaller. Alternatively, if 
we assume the blue broad component is from virialized gas around and illuminated only by the secondary, and the continuum luminosity of the secondary is one-tenth the value of total continuum (which is roughly the line flux ratio between the blue Gaussian component and the double-peaked disk line in $\mathrm{H} \alpha$ ), then following the L-R relation, the virial mass of the secondary black hole is about $M_{2}=10^{7} M_{\odot}$. Note that the black hole masses we estimated are similar to the values derived by Boroson \& Lauer $(2009)\left(M_{1}=10^{8.9} M_{\odot}, M_{2}=10^{7} .3 M_{\odot}\right)$ in a somewhat different way.

\section{DISCUSSION}

Since we wish to make as few assumptions as possible, and since both binary SMBHs and double-peaked emitters are rarely observed, why do we include both? We answer this question as follows.

1. SDSS J1536+0441 is likely a double-peaked emitter. The red shoulders in the emission lines shown in Chornock et al. (2009), and the fact that it is fit well by our simple disk model line by excluding both the blue and central peaks, suggest there are emissions from the disk.

2. There is no simple model that works well for SDSS J1536+0441. The sharp blue peak has not been seen before in any double-peaked emitter. Lauer \& Boroson (2009) listed three that may be analogous to SDSS J1536+0441, but none of them seem convincing. Therefore, if we accept the hypothesis that SDSS J1536+0441 is a double-peaked emitter, it must be a peculiar one, which then requires additional unusual assumptions.

3. The existence of a minor black hole is physically more natural than an extremely asymmetric accretion disk. In fact, we do expect SMBH binaries as natural consequences of galaxy mergers (Begelman et al. 1980; Civano et al. 2009; Comerford et al. 2009).

4. Double-peaked emission lines are not common and most $(\sim 97 \%$ at $z<0.33)$ AGNs in the SDSS sample do not show double-peaked line profiles (Strateva et al. 2003). Instead of asking why a few percent of AGNs show double-peaked emission lines, we ask why most AGNs do not show double-peaked emission lines, since virtually all AGNs must have accretion disks. Possible reasons include: i) a vertical extended ionizing structure is required for double-peaked emitters (Chen \& Halpern 1989), so if there is no illuminating source shining on the disk, the double-peaked lines will either disappear or be weak and buried on the continuum, or ii) if the disk extends to large radius (Rokaki et al. 1992; Jackson et al. 1991), iii) the disk is face-on (Corbin 1997) or iv) there is a disk wind (Murray \& Chiang 1997; Eracleous et al. 2004). Any of the above could smear out the double-peaked profile. We note that the existence of a minor black hole helps produce disk emission lines in two aspects. First, when the secondary spirals in, the outer edge of the inner disk will form a density spike and puff up (Armitage \& Natarajan 2000). Therefore the covering factor of the disk becomes larger; and a hot vertical extended torus (Chen \& Halpern 1989) or outflow might also be formed due to the disturbance brought by the minor black hole (Armitage \& Natarajan 2000), which helps to illuminate the disk. As discussed in Eracelous \& Halpern (2003), such a vertical extended structure will also help explain the preferential association of double-peaked emitters with radio-loud AGNs (as shown in Strateva et al. 2003 that double-peaked AGNs are 1.6 times more likely to be radio sources), since the original vertical extended ion torus model (Rees et al. 1982) was intended to explain the formation of radio jets. Second, when the secondary black hole approaches merger with the primary black hole, the disk is truncated at a radius of the order of the binary separation, therefore the double-peaked profile is not smeared.

Since SDSS J1536+0441 can not be unique as the only double-peaked emitter in a binary supermassive black hole system, such systems might also exist in many known double-peaked emitters, where the tidal torques from the secondary black hole clear the outer region of the disk around the primary black hole, and might also help illuminate the disk. This leads to the question: why do other double-peaked emitters not show any evidence of the existence of a secondary black hole?

There are two possible reasons. First, when the secondary is much smaller than the primary, its BLR might be totally stripped, and its heating on the edges of the disks is not strong enough to produce excess emission line components. Therefore, the emission from the accretion disk around the primary dominates. In such cases, the secondary might excite spiral wave or other instabilities in the accretion disk around the primary and cause the variability of the double-peaked emission lines, such as seen in 3C 390.3 (Chakrabarti \& Wiita 1993; Eracleous et al. 1997; Gilbert et al. 1999). Moreover, about half of the double-peaked emitters require some form of "non-axisymmetries", and how to produce the asymmetries is challenging since a significant perturbation is needed. The secondary will naturally bring asymmetries to the system. In such cases, we would also expect these double-peaked emitter AGNs to be more variable than the average, which is the case for optical X-ray variability on timescales of years (see e.g. Strateva et al. 2008). Second, the reason why we haven't identified any double-peaked emitter binary system yet is in order to find the signature of a secondary black hole, we need long-term observations, which are generally not available (but could be from the Digital Access to a Sky Century @ Harvard (DASCH) project - see Grindlay et al. 2009). Several double-peaked emitters have been monitored for two decades (Halpern \& Filippenko 1988; Eracleous et al. 1997; Gezari et al. 2007 and references therein), and in most of them, there is no evidence for the systematic drift signal from a binary system. However, in one system, Arp 102B, which is the prototype disk emitter, there is an excess component which oscillated between the red and blue peaks of the disk line from 1991 to 1994, and from 1999 to 2003 (Newman et al. 1997; Gezari et al. 2007). During both epochs, the period is about 2 yrs, and the velocity drift amplitude is about $8000 \mathrm{~km} / \mathrm{s}$ from 1991 to 1994, and about $6000 \mathrm{~km} / \mathrm{s}$ from 1999 to 2003. Therefore, if both oscillations are caused by an orbiting bright spot, the two derived masses of the central SMBH are discrepant by a factor of 2 (Gezari et al. 2007). We suggest that it could be a SMBH binary, and the periodic drift excess components are from the heated rims caused by the orbital motion of the secondary, while the contributions from the inner and outer rims are different in the two epochs. The transient nature of the excess could be due to the instabilities in the system (Lodato 
et al. 2009). The flux ratio from the two rims likely also changes over a timescale of several orbits. Therefore the derived amplitude of the velocity of the excess component also changes. Here the oscillation period could be the orbital period of the secondary, the velocity drift from 1991 to 1994 could be dominated by the orbital motion of the heated inner rim near the secondary, and the velocity drift from 1999 to 2003 could be dominated by the orbital motion of the heated outer rim near the secondary which has relatively smaller amplitude.

Another question is, are all binary black hole AGN systems double-peaked emitters? Our answer is they are not. The stage that both black holes have BLRs is likely to be short lived. The inner disk around the primary might be depleted soon since the secondary acts like a dam and the matter in the outer circum-binary disk can hardly get through the gap swept by the secondary (Lodato et al. 2009; but see Artymowicz \& Lubow 1996 for gas streams penetrating the disk gap and supply mass to the binary). A similar SMBH binary model has been proposed by Bogdanovic et al. (2009) to explain another multiple-redshifts system, SDSS J092712.65+294344.0 (hereafter SDSS J0927). SDSS J0927 was discovered by Komossa et al. (2008) as a candidate for a recoiling black hole, and is the other system found in the search by Boroson \& Lauer (2009), which shows two sets of narrow lines and only the blue one has associated broad lines. Bogdanovic et al. (2009) proposed that the system is a SMBH binary surrounded by a circum-binary disk (Ivanov et al. 1999; Cuadra et al. 2009), where the primary is inactive and has no BLR, while the secondary moving towards us is accreting and emits broad lines from its accretion disk and narrow lines from the inner rim of the circum-binary disk, which might resemble the later evolutionary stage of SDSS J1536+0441 in our model.

If we adopt $M_{1}=10^{9} M_{\odot}, M_{2}=10^{7} M_{\odot}$, and a Keplerian projected velocity $3500 \mathrm{~km} / \mathrm{s}$ of the secondary at inclination $47^{\circ}$, then the separation of the two black holes is $0.38 \mathrm{pc}$ and the period is $490 \mathrm{yr}$. The decay timescale for the binary to merge due to gravitational radiation alone is $1 \times 10^{12} \mathrm{yr}$ (Peters 1964). However, the gas disk around the black holes will help solve the so called final parsec problem, but the timescale given in hydrodynamic simulations is quite uncertain and depends on the mass ratio between the disk and the secondary (Lodato et al. 2009; Cuadra et al. 2009). If we assume that all double-peaked emitters are merging binary systems in the gas dissipation stages, then there will be $\geq 3 \%$ nearby AGNs in merging binary systems. This means, on average, these AGNs spend $\geq 4 \times 10^{8}$ years in merging. If there is one merger event per lifetime, then each merger takes $\geq 4 \times 10^{8}$ years in its gas dissipation phase, which is roughly a few times the disk's viscous timescale, and so consistent with the hydrodynamic simulation re- sults when the disk mass is larger than or comparable with the secondary mass (Armitage \& Natarajan 2002; Lodato et al. 2009).

For our estimated 490 years for the orbital period of SDSS J1536+0441, we may not be able to see the velocity change of the blue peak over several years. Spectroscopic monitoring on timescales of decades could provide the most direct test of the binary hypothesis. A possible test of our model in the near future might be UV observations from refurbished HST. As discussed in Chornock et al. (2009), the Ly $\alpha$ and C IV lines in double-peaked emitters frequently lack double-peaked velocity profiles (Halpern et al. 1996; Eracleous et al. 2004). Eracleous et al. (2004) suggested that the double-peaked low-ionization Balmer lines are from the dense accretion disk where the Ly $\alpha$ emission is suppressed, and the single-peaked Ly $\alpha$ line is from a lower density higher-ionization wind. So under the picture of Eracleous et al. (2004), there are three possible cases:

1. If the blue peaks in the $\mathrm{H} \alpha$ and $\mathrm{H} \beta$ lines in SDSS $\mathrm{J} 1536+0441$ are from the accretion disk of a single SMBH (as in Chornock et al. 2009), then a blue peak is not likely to be present in the Ly $\alpha$ profile;

2. If the blue peaks in the $\mathrm{H} \alpha$ and $\mathrm{H} \beta$ lines in SDSS $\mathrm{J} 1536+0441$ are from region around the secondary $\mathrm{SMBH}$, i.e. either accretion flow around the secondary or rims of the inner circum-primary and outer circum-binary disks, but there is no lower density, higher-ionization wind from the secondary, then a blue peak is not likely to be present in the Ly $\alpha$ profile;

3. If the blue peaks in the $\mathrm{H} \alpha$ and $\mathrm{H} \beta$ lines in SDSS J1536+0441 are from region around the secondary SMBH, and there is a lower density, higherionization wind from the secondary (possibly arisen from the accretion disk around the secondary), then a blue peak will be present in the Ly $\alpha$ profile.

Therefore, the presence of blue peaks shifted by $\sim 3500$ $\mathrm{km} / \mathrm{s}$ in the Ly $\alpha$ and C IV emission lines would strongly support our model (case 3); the absence of multiple velocity peaks in the Ly $\alpha$ and C IV emission lines, however, would still leave the question open (neither case 1 nor case 2 could be ruled out).

We are grateful to the anonymous referee for helpful comments and suggestions. S.T. thanks Martin Elvis, Shuang Nan Zhang, Yuexing Li for stimulating discussions and Lei Hao for valuable suggestions. We thank Ryan Chornock for sending us the spectra data in their paper.

\section{REFERENCES}

Armitage, J. E., \& Natarajan, P. 2002, ApJ, 567, L9

Artymowicz, P., \& Lubow, S. H. 1996, ApJ, 467, L77

Baldwin, J. A., Ferland, G. J., Korista, K. T., Hamann, F., \& LaCluyze, A. 2004, ApJ, 615, 610

Begelman, M. C., Blandford, R. D., \& Rees, M. J. 1980, Nature, 287, 307

Bogdanovic, T., Eracleous, M., \& Sigurdsson, S. 2009, ApJ, 697, 288
Boroson, T. A., \& Lauer, T. R. 2009, Nature, 458, 53

Brotherton, M. S., Wills, B. J., Francis, P. J., \& Steidel, C. C. 1994, ApJ, 430, 495

Chakrabarti, S. \& Wiita, P. J. 1993, ApJ, 411, 602

Chen, K., Halpern, J. P. 1989, ApJ, 344, 115

Chen, K., Halpern, J. P., \& Filippenko, A. V. 1989, ApJ, 339, 742

Chornock, R., et al. 2009, ApJ, submitted, arXiv:0906.0849

Civano, F., et al. 2009, ApJ, submitted 
Corbin, M. 1997, ApJ, 485, 517

Cuadra, J., Armitage, P. J., Alexander, R. D., \& Begelman, M. C. 2009, MNRAS, 393, 1423

Collin-Souffrin, S., \& Dumont, A. M. 1989, A\&A, 213, 29

Comerford, J. M. et al. 2009, ApJL, submitted, arXiv:0906.3715

Corbin, M. A. 1997, ApJ, 485, 517

Decarli, R. et al. 2009, ApJ, in press, arXiv:0907.5414

Dumont, A. M., \& Collin-Souffrin, S. 1990, A\&A, 229, 313

Elvis M. 2000, ApJ, 545, 63

Eracleous, M., \& Halpern, J. P. 1994, ApJS, 90, 1

Eracleous, M., \& Halpern, J. P. 2003, ApJ, 599, 886

Eracleous, M., Halpern, J. P., Gilbert, A. M., Newman, J. A., \& Filippenko, A. V. 1997, ApJ, 490, 216

Eracleous, M., Halpern, J. P., Storchi-Bergmann, T., Filippenko, A. V., Wilson, A. S., \& Livio, M. 2004, in The Interplay Among Black Holes, Stars and ISM in Galactic Nuclei, ed. T. Storchi- Bergmann, L. C. Ho, \& H. R. Schmitt (Cambridge: Cambridge Univ. Press), 29

Gaskell, C. M. 2009, ArXiv e-prints, arXiv:0903.4447

Gaskell, C. M. 1983, in Proc. 24th Liege Int. Astrophys. Colloq., Quasars and Gravitational Lenses (Cointe-Ougree: Univ. Liege), 471

Gezari, S., Halpern, J. P., \& Eracleous, M. 2007, ApJS, 169, 167

Gilbert, A. M., Eracleous, M., Filippenko, A. V., \& Halpern, J. P. 1999, in ASP Conf. Ser. 175, Structure and Kinematics of Quasar Broad Line Regions, ed. C. M. Gaskell,W. N. Brandt,M. Dietrich, D. Dultzin-Hacyan,\&M. Eracleous (San Francisco: ASP), 189

Grindlay, J., Tang, S., Simcoe, R., Laycock, S., Los, E., Mink, D., Doane, A., \& Champine G. 2009, in preparation

Gould, A., \& Rix, H.-W. 2000, ApJ, 532, L29

Halpern, J. P., Eracleous, M., Filippenko, A. V., \& Chen, K. 1996, ApJ, 464, 704

Halpern, J. P. \& Filippenko, J. P. 1988, Nature, 331, 46

$\mathrm{Hu}, \mathrm{C}$. et al. 2008a, ApJ, 683, L115

$\mathrm{Hu}, \mathrm{C}$. et al. 2008b, ApJ, 687, 78

Ivanov, P. B., Papaloizou, J. C. B., \& Polnarev, A. G. 1999, MNRAS, 307, 79
Jackson, N., Penston, M. V., \& Perez, E. 1991, MNRAS, 249, 577

Kaspi, S., Maoz, D., Netzer, H., Peterson, B. M., Vestergaard, M., \& Jannuzi, B. T., 2005, ApJ, 629, 61

Kaspi, S., Smith, P. S., Netzer, H., Maoz, D., Jannuzi, B. T., \& Giveon, U. 2000, ApJ, 533, 631

Komossa, S., Zhou, H., \& Lu, H. 2008, ApJ, 678, L81

Lauer, T. R., \& Boroson, T. A. 2009, ApJ, submitted, arXiv:0906.0020

Lewis, K., \& Eracleous, M. 2006, ApJ, 642, 711

Lodato, G., Nayakshin, S., King, A. R., \& Pringle, J. E. 2009, MNRAS, in press, arXiv:0906.0737

Miyoshi, M., et al. 1995, Nature, 373, 127

Murray., N., \& Chiang, J. 1997, ApJ, 474, 91

Newman, J. A., Eracleous, M., Filippenko, A. V., \& Halpern, J. P. 1997, ApJ, 485, 570

Peters, P. C. 1964, Phys. Rev., 136, 1224

Peterson, B. M. et al., 2004, ApJ, 613, 682

Rees, M. J., Begelman, M. C., Blandford, R. D., \& Phinney, E. S. 1982, Nature, 295, 17

Rokaki, E., Boisson, C., \& Collin-Souffrin, S. 1992, A\&A, 253, 57

Schneider, D. P., et al. 2007, AJ, 134, 102

Stirpe, G. M. 1990, A\&AS, 85, 1049

Strateva, I. V., et al. 2003, AJ, 126, 1720

Strateva, I. V. et al. 2006, ApJ, 651, 749

Strateva, I. V., Brandt, W. N., Eracleous, M., \& Garmire, G. 2008, ApJ, 687, 869

Vestergaard, M. \& Peterson, B. M. 2005, ApJ, 625, 688

Wrobel, J. M., \& Laor, A. 2009, ApJ, in press, arXiv:0905.3566

Wu X.-B., \& Liu F.-K., 2004, ApJ, 614, 91

Young, P. \& Schneider, D. P. 1980, ApJ, 238, 955

Zhang, X.-G., Dultzin-Hacyan, D., \& Wang, T.-G. 2007, MNRAS, 376, 1335

Zhu, L., Zhang, S. N., Tang, S. 2009, ApJ, in press, arXiv:0807.3992 